\title{
Cooperation in Diffusive Spatial Games
}

\author{
Mendeli H. Vainstein*, Ana T. C. Silva ${ }^{\dagger}$ and Jeferson J. Arenzon** \\ ${ }^{*}$ Instituto de Fisica and International Center of Condensed Matter Physics, Universidade de Brasília, CP \\ 04513, 70919-97, Brasilia, DF, Brazil \\ ${ }^{\dagger}$ Departamento de Física, Universidade Estadual de Feira de Santana, Campus Universitário, $\mathrm{km} 3, \mathrm{BR} 116$, \\ 44031-460 Feira de Santana BA, Brazil \\ ${ }^{* *}$ Instituto de Fisica, Universidade Federal do Rio Grande do Sul, CP 15051, 91501-970 Porto Alegre RS, Brazil
}

\begin{abstract}
Random diffusion is shown to be an important mechanism on fostering cooperative behavior among simple agents (memoryless, unconditional cooperators or defectors) living on a spatially structured environment. In particular, under the Prisoner's Dilemma framework, when allowing the agents to move with the simple "always-move" rule, we find that cooperative behavior is not only possible but may even be enhanced. In addition, for a broad range of densities, mobile cooperators can more easily invade a population of mobile defectors, when compared with the fully viscous, immobile case. Thus, such simple mobility pattern may have played a fundamental role both in the onset and development of cooperative behavior, paving the way to more complex, individual and group, motility rules.
\end{abstract}

Keywords: Game theory, Prisoner's Dilemma, Cooperation, Diffusion

PACS: $87.23 . \mathrm{Ge}$

Among the top 25 scientific questions listed on a recent issue of the Science magazine (July 1, 2005), representative of many major gaps in our basic knowledge, there was "How did cooperative behavior evolve?". For a long time, this question has been eluding scientists coming from very different fields, ranging from psychological and social sciences to statistical physics, even though remarkable progress and insights have been accumulated in the last decades $[1,2,3,4,5,6]$. Several mechanisms have been identified, like direct, indirect and spatial reciprocity, kinship and group selection, but a complete answer regarding the general conditions under which cooperative behavior may evolve and be sustained is still lacking [7]. Cooperation, although involving a cost to the performer, is ubiquitous in biological populations, even in groups of extremely simple individuals $[8,9,10,11,12,13,14,15,16,17,18]$ where a mechanism not relying on complex memory functions or cognitive processes must be invoked. One such mechanism is spatial or network reciprocity: when dispersal is very limited (high population viscosity), differently from the standard, random mixing population, the continuing interaction within the local neighborhood increases the probability of future encounters and the maintenance of cooperation. To model such a situation, Nowak and May [19, 20] extended the first considerations on the effects of territoriality in the spread of strategies in the Prisoner's Dilemma game done by Axelrod [2], and showed that with geographical fixation, even simple nice rules like unconditional cooperation are able to survive. In these structured populations, cooperative strategies can build clusters in which the benefits of mutual cooperation can outweigh losses against defectors, maintaining the population of cooperators stable. These spatial games, in which the interactions are localized and non random, have been studied and extended in many ways (see, for example, Refs. [21, 22, 23, 24, 25, 26, 27, 28, 29, 30, 31, 32, 33, 34, 35, 36, 37, 38, 39, 40, 41, 42, 43, 44, 45]). In such spatially structured populations, one may wonder about the effects of mobility: is it possible to evolve and sustain cooperation in a population of mobile agents? In particular, is it possible to have a finite density of unconditional cooperators with unbiased, random mobility? Once mobile, cooperators can move away from the supporting clusters of other cooperators, becoming targets for exploitation, while defectors, on their turn, can exploit cooperators and move away to avoid retaliation. Moreover, mobility helps to increase the random mixing and it is known that when every agent interacts randomly with the whole population, defection prevails. With such considerations, it came as a surprise [46] that even a blind pattern of mobility, without anticipating the future neighborhood and without considering the accumulated payoff, may have a positive effect in the amount of cooperation, and not be a limiting factor for it.

Many previous works have considered the role of diffusion in the problem of cooperation $[47,48,49,50,51,52$, $53,54,36,55,56]$. The present work, which complements the discussion of Ref. [46], differs from all these in several important aspects: we consider non random interactions on a two-dimensional structure, mobility traits do not evolve and individual movements are Brownian, non contingent, and not under the control of the agents, both strategies

CP913, Nonequilibrium Statistical Mechanics and Nonlinear Physics, XV Conference

edited by O. Descalzi, O. A. Rosso, and H. A. Larrondo

C) 2007 American Institute of Physics 978-0-7354-0421-2/07/\$23.00 

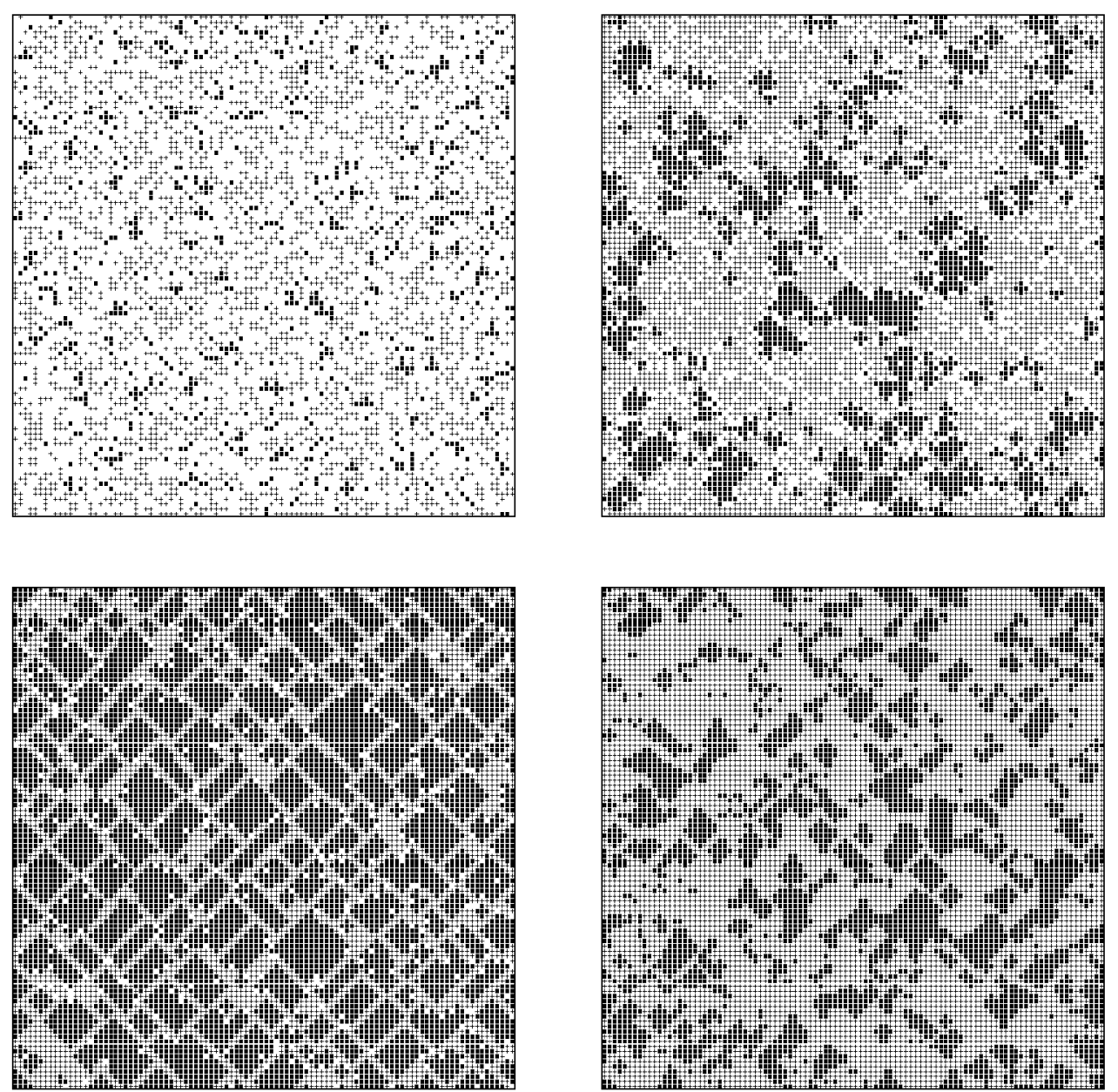

FIGURE 1. Snapshots of typical configurations once the asymptotic state has been reached for a system with no mobility $(m=0)$ and different densities, from top to bottom, left to right: $\rho=0.4,0.8,0.962$ (this corresponds to the optimal density for cooperation) and 1. All simulations are performed on a square lattice of side length $L=100, b=1.4$, and cooperators (defectors) are represented by squares (crosses). Vacant sites are painted white. In the top left panel, cooperators may survive either because they are isolated or belong to a weakly predated cluster. The remaining panels present high density situations, well above the percolation threshold (a spanning cluster is present), with quite different cluster structures in each case. Notice that once the system percolates, the spanning cluster is formed by defectors, even when they are a minority (like in the bottom left panel).

considered are simple, unconditional and non retaliating, with no memory of previous steps. In other words, we are considering a basic scenario, whose importance is to settle the minimal conditions under which cooperative behavior might be sustained once the requirement of strong viscosity for spatial reciprocity is relaxed. In particular, we show that in the framework of the spatial Prisoner's Dilemma (PD) game under the above conditions, cooperative behavior, besides being robust, can even be diffusion-enhanced.

In any round of the PD game, each of two players either cooperates (C) or defects (D). Here we only consider pure strategies, although more complex rules, with memory of previous encounters, have been devised [2]. The payoff depends on the mutual choice and is given by the matrix whose elements are: a reward $R$ (punishment $P$ ) if both cooperate (defect), $S$ (sucker's payoff) and $T$ (temptation) if one cooperates and the other defects, respectively. Moreover, these quantities should satisfy the inequalities $T>R>P>S$ and $2 R>T+S$. Here we will take a simplified, one parameter version [19]: $R=1, P=S=0$ and $T=b>1$. In a random mating, infinite population defecting will be the most rewarding strategy, independently of the opponent's choice. On a network of contacts, each individual 


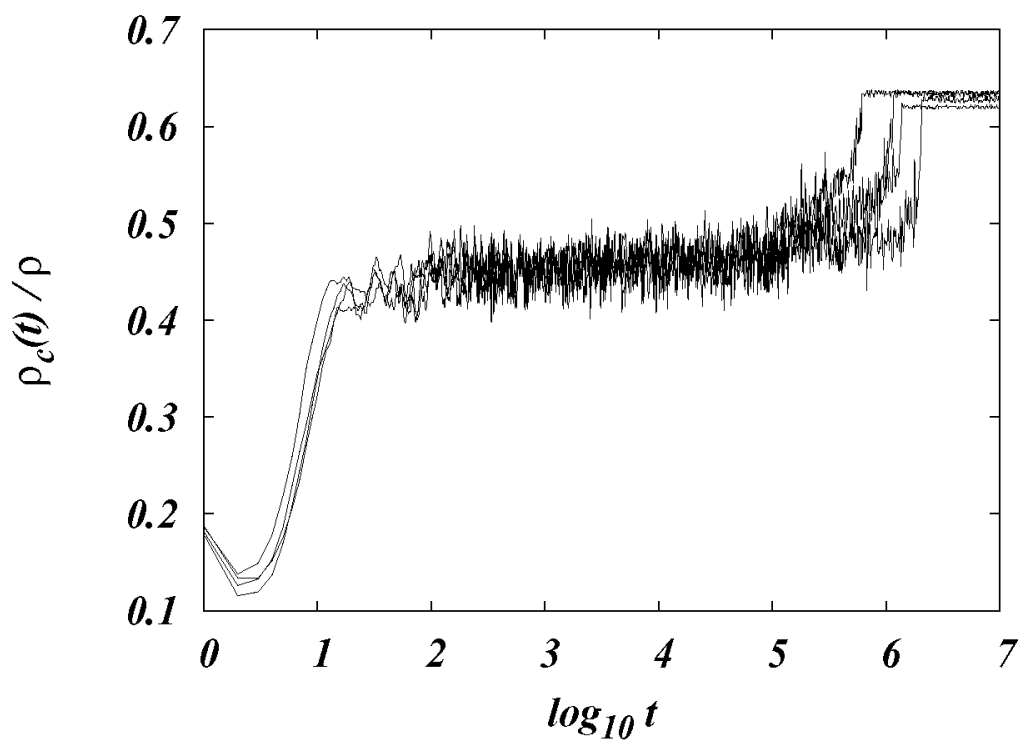

FIGURE 2. Time evolution of cooperator density at the point where their frequency in the population is maximum. Due to the presence of quenched disorder, it evolves very slowly and after many decades it jumps to the equilibrium value. Different lines represent several samples.

combats with its closest neighbors (if any) and accumulates the corresponding payoff, that will be compared, during the offspring step, with the payoff accumulated by the neighbors.

We addressed in an earlier work [31] the question of the robustness of cooperation in spatial games in the presence of heterogeneous environments. By introducing quenched disorder in the lattice (random dilution) each individual would sense a locally varying social environment as the number of neighbors becomes site dependent, and optimal cooperation is achieved for weak disorder as the defects (or inaccessible regions) act as pinning fields, keeping the clusters of cooperators more protected from invasions. In this sense, an irregular landscape may enhance cooperation by introducing natural defenses against invasions of defectors. In fig. 1 we present several snapshots for the case where there are no mobile agents and different lattice occupations. When the density is low, the clusters are disconnected and the fate of each isolated cluster depends on the initial distribution of cooperators and defectors: cooperation is guaranteed by geographical isolation. As density increases, the system percolates. Interestingly, the spanning cluster is always built by defectors, while cooperators tend to organize in more compact groups, what is essential for their stability. Cooperation is maximized for weak disorder $(\rho \simeq 0.962)$ and it is the pinning effect of the empty sites that drives the enhancement in the amount of cooperators. Close to this value, the cluster geometry is also dramatically changed as can be observed in the figure. Moreover, the presence of disorder also has a remarkable effect in the way the system evolves, as can be seen in figure 2, where the time evolution of five samples whose density is very close to the critical occupation is shown. After a fast transient there is a very slow increase of the number of cooperators that lasts several decades, followed by an abrupt, avalanche-like jump to the stationary state value. Such slow dynamics is a general feature of systems with quenched disorder and the average jumping time has a very fast increase as the lattice occupation approaches $\rho \simeq 0.962$.

When the vacant sites are no longer held fixed, they may become occupied by a neighbor agent with a probability $m$ that measures the populational viscosity. Only random, unbiased diffusion has been considered in Ref. [46], although extensions to contingent rules are also being studied. As before, individuals combat with their closest neighbors and accumulate a given payoff. Then, they may either move or try to generate their offspring, following the player with the greatest payoff in the neighborhood. The detailed outcome of the game will depend on the precise implementation of the dynamics, in particular, the order in which combats, offspring generation and diffusion occur leads to qualitative and quantitative differences in the population. As an example, in fig. 3 the asymptotic state for a density far above the percolation threshold is shown for two dynamics where the order of the diffusive and offspring steps are reversed (Combat-Offspring-Diffusion, COD, and Combat-Diffusion-Offspring, CDO, dynamics). In the former, as the name says, each step consists of combats followed by the generation of offspring done in parallel, and then diffusion, while 

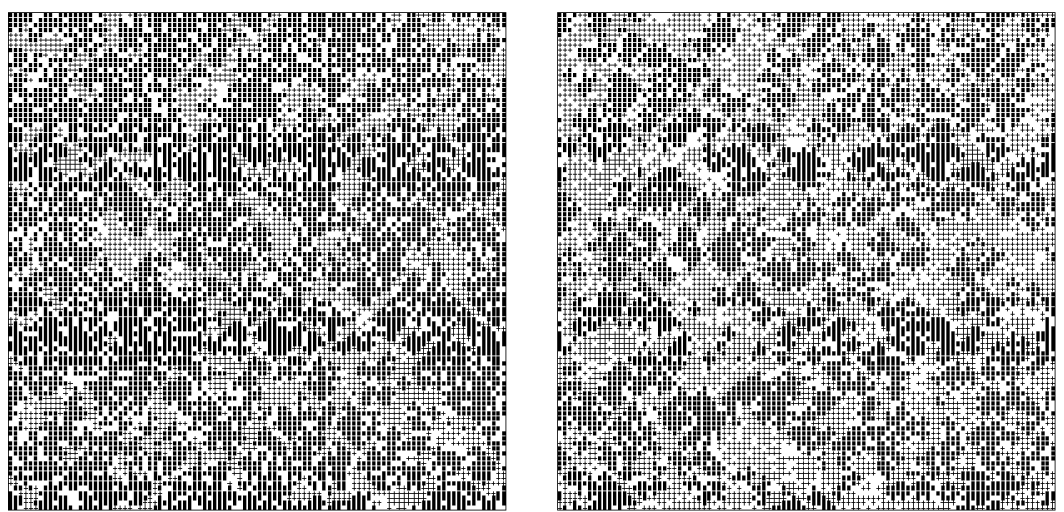

FIGURE 3. Snapshot of typical configurations after the asymptotic state has been achieved for a system with $\rho=0.8$ and $m=1$, for CDO (left) and COD dynamics (right). Empty sites are left blank, while cooperators and defectors are represented by squares and crosses, respectively. Compare with the corresponding snapshot in Fig. 1 where $m=0$.
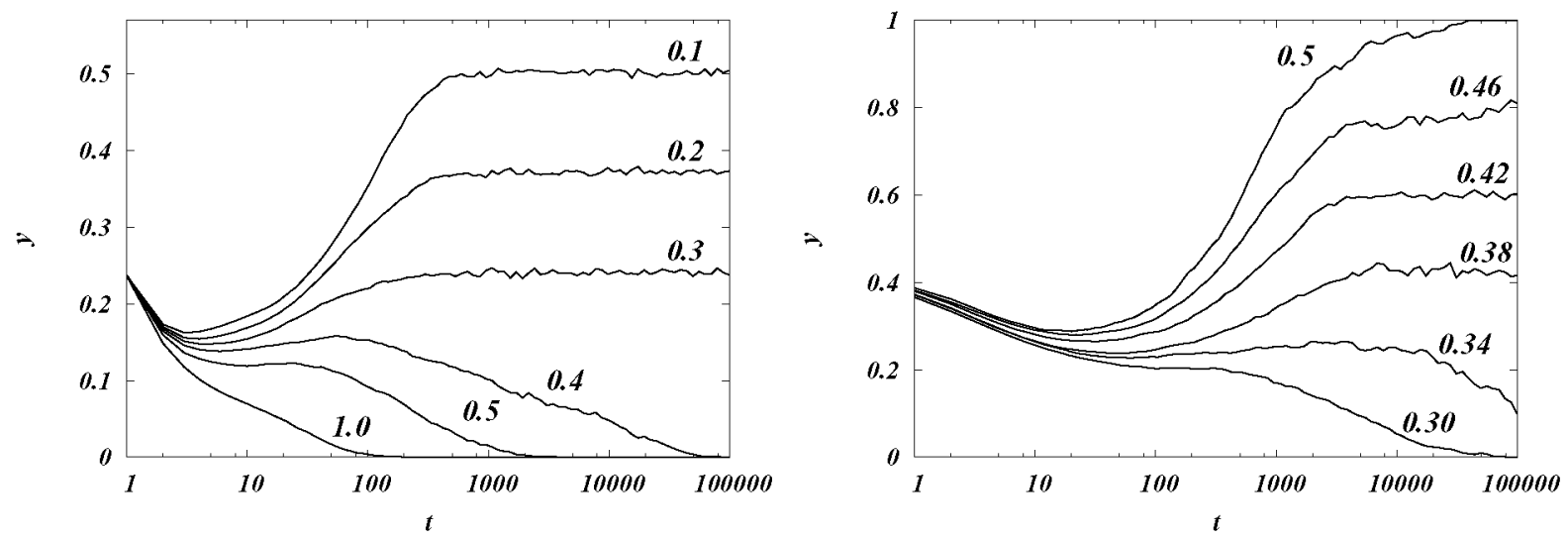

FIGURE 4. Average fraction of cooperators $\rho_{\mathrm{c}} / \rho$ as a function of time for several values of $m$ for the COD dynamics at $\rho=0.7$ (left) and $\rho=0.24$ for the CDO dynamics (right). In all cases there is an initial decrease in the cooperator density since cooperators are not yet coordinated and only form small groups, being easily predated. Depending on the mobility, at later times the existing cooperator clusters may either disappear or grow.

in the later, the diffusion and offspring steps are reversed. During the diffusive step, each agent makes an attempt to jump to an empty neighboring site, what is accepted with a probability $m$.

Fig. 4, with the temporal evolution of the average cooperator density $\rho_{\mathrm{c}}$, exemplifies the rich behavior presented by the model once mobility is introduced. The ultimate fate of cooperation depends on the population viscosity $m$, its total density and probably on the initial state: while in the COD dynamics (Fig. 4, left) the asymptotic density of cooperators decreases as $m$ increases, in the CDO case (Fig. 4, right), on the contrary, $\rho_{\mathrm{c}}$ may increase with $m$ for some values of $\rho$. The detailed dependence on the mobility and density of the asymptotic number of cooperators was studied in Ref. [46], and it was shown that for broad conditions, cooperation is enhanced when compared with the viscous case of $m=0$. This effect is even stronger in the CDO case: whenever $m \neq 0$, it is good for the cooperators to move away from their partner, whatever its strategy, after the combats and before the payoff comparison of the offspring step, what favors the CDO dynamics. In this way, an exploited cooperator can still leave descendants if, after diffusing, it is not neighbored by a high payoff defector. Another important effect is that mobility facilitates the onset of cooperation: as shown in Ref. [46], starting with an initial patch with only two cooperators in a sea of defectors, the probability that cooperation overtakes the whole population is an increasing function of the mobility for a wide range of densities. In comparison, this has a very small probability of happening when $m \simeq 0$. 
A possible realization of such diffusion scenario may occur in organisms with extracellular metabolism, as is the case of some yeast cells [15]. Sugar is processed outside the cell by a secreted enzyme called invertase, creating a common resource for all surrounding cells. This offers the opportunity for defection as some cells may not have the cost of producing the enzyme but yet benefit from that produced by others. To what extent the cooperative behavior observed in such simple organisms is a sole effect of the underlying spatial structure or whether there is an enhancement factor due to diffusion is an open and interesting question. Moreover, in systems that present polarized motion (chemotaxis), depending on the concentration of cooperators, a gradient of nutrients may be present and both cooperators and defectors can migrate towards (away) high cooperator (defector) density regions. Thus, a possible rule in this case can be: "cooperators attract-defectors repel", but many others can be devised. Such non random rules may also develop cooperative swarming or foraging, relevant for evolving higher levels of biological organization, and is already found to occur in bacteria [13].

Here we presented results for a simple spatial game where the patched environment allows explicit, although random, movement of agents whose strategies are pure, non retaliating (unconditional). The diffusion is Brownian, not relying on any type of explicit, genotypic or phenotypic assortment. Whether a given strategy is able or not to invade another population would strongly depend on how viscous a population is, the global density and the chosen dynamics. However, some universal conclusions can be stated. First of all, cooperation is possible under the above conditions, thus enlarging the limits for cooperative behavior. Second, for a broad range of the parameters (density, viscosity, etc), cooperation is enhanced in respect to the viscous case. Third, a rule like always-move, regardless of the opponent strategy, may increase the capability of cooperators to invade and overtake a population of defectors. In this sense, mobility may have a fundamental role in the problem of the onset of cooperation, and the evolution of a simple mobility as the one studied here, is a first step towards the emergence of more complex foraging behavior or even coherent, cooperative herding behavior.

\section{ACKNOWLEDGMENTS}

This work was partially supported by the Brazilian agencies CAPES, CNPq and FAPERGS.

\section{REFERENCES}

1. J. Maynard Smith, Evolution and the Theory of Games, Cambridge University Press, Cambridge, UK, 1982.

2. R. Axelrod, The Evolution of Cooperation, BasicBooks, New York, 1984.

3. L. A. Dugatkin, and H. K. Reeve, Game Theory and Animal Behavior, Oxford University Press, New York, 1998.

4. C. Hauert, and G. Szabó, Am. J. Phys. 73, 405-414 (2005).

5. G. Szabó, and G. Fath, Evolutionary games on graphs (2006), cond-mat/0607344.

6. M. A. Nowak, Evolutionary Dynamics: Exploring the Equations of Life, Belknap Press, 2006.

7. M. A. Nowak, Science 314, 1560-1563 (2006).

8. P. E. Turner, and L. Chao, Nature 398, 441-443 (1999).

9. B. J. Crespi, Trends Ecol. Evol. 16, 178-183 (2001).

10. M. Vulic, and R. Kolter, Genetics 158, 519-526 (2001).

11. T. Frick, and S. Schuster, Naturwissenschaften 90, 327-331 (2003).

12. P. B. Rainey, and K. Rainey, Nature 425, 72-74 (2003).

13. G. J. Velicer, and Y. N. Yu, Nature 425, 75-78 (2003).

14. A. S. Griffin, S. A. West, and A. Buckling, Nature 430, 1024-1027 (2004).

15. D. Greig, and M. Travisano, Proc. R. Soc. London B (Suppl.) 271, S25-S26 (2004).

16. D. M. Wolf, V. V. Vazirani, and A. P. Arkin, J. Theor. Biol. 234, 255-262 (2005).

17. F. Fiegna, Y.-T. N. Yu, S. V. Kadam, and G. J. Velicer, Nature 441, 310-314 (2006).

18. N. J. Mehdiabadi, C. N. Jack, T. T. Farnham, T. G. Platt, S. E. Kalla, G. Shaulsky, D. C. Queller, and J. E. Strassmann, Nature 442, 881-882(2006).

19. M. A. Nowak, and R. M. May, Nature 246, 15-18 (1992).

20. M. Nowak, and R. May, Int. J. of Bifurcation and Chaos 3, 35-78 (1993).

21. M. A. Nowak, S. Bonhoeffer, and R. M. May, Int. J. of Bifurcation and Chaos 4, 33-56 (1994).

22. M. A. Nowak, S. Bonhoeffer, and R. M. May, Proc. Natl. Acad. Sci. USA 91, 4877-4881 (1994).

23. K. Lindgren, and M. G. Nordahl, Physica D 75, 292-309 (1994).

24. P. Grim, J. Teor. Biol. 173, 353-359(1995).

25. T. Killingback, and M. Doebeli, Proc. R. Soc. Lond. B 263, 1135-1 144 (1996).

26. M. Nakamaru, H. Matsuda, and Y. Iwasa, J. Theor. Biol. 184, 65-81 (1997). 
27. G. Szabó, and C. Töke, Phys. Rev. E 58, 69-73 (1998).

28. K. Brauchli, T. Killingback, and M. Doebeli, J. Theor. Biol. 200, 405-417 (1999).

29. T. Killingback, M. Doebeli, and N. Knowlton, Proc. R. Soc. B 266, 1723-1728 (1999).

30. G. Szabó, T. Antal, P. Szabó, and M. Droz, Phys. Rev. E 62, 1095-1103 (2000).

31. M. H. Vainstein, and J. J. Arenzon, Phys. Rev. E 64, 051905 (2001).

32. G. Abramson, and M. Kuperman, Phys. Rev. E 63, 030901 (2001).

33. C. Hauert, Int. J. Bif. Chaos 12, 1531-1548 (2002).

34. B. Kim, A. Trusina, P. Holme, P. Minnhagen, J. Chung, and M. Choi, Phys. Rev. E 66, 021907 (2002).

35. J. Miekisz, J. Phys. A: Math. Gen. 37, 9891-9906 (2004).

36. C. A. Aktipis, J. Theor. Biol. 231, 249-260 (2004).

37. C. Hauert, and M. Doebeli, Nature 428, 643-646 (2004).

38. H. Fort, and S. Viola, J. Stat. Mech.: Teor. Exp. 01, P01010 (2005).

39. F. C. Santos, and J. M. Pacheco, Phys. Rev. Lett. 95, 098104 (2005).

40. O. Durán, and R. Mulet, Physica D 208, 257-265 (2005).

41. V. M. Eguiluz, M. G. Zimmermann, C. J. Cela-Conde, and M. San Miguel, Am. J. Sociol. 110, 977-1008 (2005).

42. R. O. S. Soares, and A. S. Martinez, Physica A 369, 823-829 (2006).

43. F. C. Santos, J. M. Pacheco, and T. Lenaerts, Proc. Natl. Acad. Sci. 103, 3490-3494 (2006).

44. R. A. Hammond, and R. Axelrod, Theor. Pop. Biol. 69, 333-338 (2006).

45. C. Hauert, J. Theor. Biol. 240, 627-636(2006).

46. M. H. Vainstein, A. T. C. Silva, and J. J. Arenzon, J. Theor. Biol. 244, 722-728 (2007).

47. L. A. Dugatkin, and D. S. Wilson, American Naturalist 138, 687-701 (1991).

48. D. S. Wilson, G. B. Pollock, and L. A. Dugatkin, Evol. Ecol. 6, 331-341 (1992).

49. P. D. Taylor, Evol. Ecol. 6, 352-356 (1992).

50. M. Enquist, and O. Leimar, Anim. Behav. 45, 747-757 (1993).

51. R. Ferrière, and R. E. Michod, Proc. R. Soc. B 259, 77-83 (1995).

52. R. Ferrière, and R. E. Michod, Am. Nat. 147, 692-717 (1996).

53. M. van Baalen, and D. A. Rand, J. theor. Biol. 193, 631-648 (1998).

54. J. C. Koella, Proc. R. Soc. B 267, 1979-1985 (2000).

55. I. M. Hamilton, and M. Taborsky, Proc. R. Soc. B 272, 2259-2267 (2005).

56. J.-F. Le Galliard, F. Ferrière, and U. Dieckmann, Am. Nat. 165, 206-224 (2005). 
Copyright of AIP Conference Proceedings is the property of American Institute of Physics and its content may not be copied or emailed to multiple sites or posted to a listserv without the copyright holder's express written permission. However, users may print, download, or email articles for individual use. 